\title{
Influence of paroxetine and cognitive/behavioral strategies in neurocardiogenic syncope and depression: a case report
}

\author{
Reg Arthur Williams ${ }^{*}$, Juan F. Lopez ${ }^{2}$ \\ ${ }^{1}$ School of Nursing and Psychiatry, Medical School, University of Michigan, Ann Arbor, USA; \\ ${ }^{2}$ Psychiatry, Medical School, University of Michigan, Ann Arbor, USA. \\ Email: rawill@umich.edu
}

Received 21 June 2011; revised 15 July 2011; accepted 24 July 2011.

\section{ABSTRACT}

Objective: Neurocardiogenic syncope (NCS) is a condition where the patient has a temporary loss of consciousness or feelings of weakness and fatigue. There are triggers such as prolonged sitting or standing, pain, and heavy exercise, but often episodes are random. Treatments are limited and the use of specific serotonin reuptake inhibitors (SSRI) have had mixed results, but a limited number of studies have suggested that paroxetine may be effective in improving the symptoms of NCS. Methods: This is a single case report of a 20 year old female who was diagnosed with NCS by a tilt test and treated conservatively with increased fluid and salt intake, and counter-pressure maneuvers. She was given one dose of sertraline, but immediately experienced disturbing visual images. She presented at the Depression Center with moderate depressive symptoms and was started on paroxetine and given cognitive/behavioral strategies to manage the NCS. Results: Since the patient had a negative experience with a prior SSRI, she was started on a low dose of paroxetine and omega-3 fatty acids. She also was given a detailed explanation of NCS and a number of cognitive/behavioral strategies such as deep breathing, progressive relaxation, imagery, and sleep. Conclusion: After 2 weeks of the multi-faceted treatment approach, she had a significant decrease in her depressive symptoms. After 6 months, the patient had no episodes of syncope and no depressive symptoms. She was able to stand for long periods and exercise without feelings of weakness and fatigue. A multimodal approach may offer the best treatment strategy to achieve full remission in patients with NCS.

Keywords: Neurocardiogenic; Paroxetine; Depression; Cognitive/Behavioral; Syncope; Case Report

\section{INTRODUCTION}

Neurocardiogenic syncope (NCS) is a condition often associated with a temporary loss of consciousness or feeling of weakness and fatigue. There is an abrupt drop in arterial blood pressure and followed rapidly with bradycardia. Often vasovagal syncope (VS) and neurally mediated syncope are terms used interchangeably with NCS [1,2]. The incident of NCS has been estimated to range from 1.3 to 2.7 events per thousand of the population $[3,4]$. Common symptoms associated with NCS, which usually occur while standing, include fatigue, weakness, nausea, perspiring, pallor, visual disturbances, abdominal discomfort, headache, tingling, lightheadedness or vertigo [5]. It is usually triggered by prolonged sitting or standing, pain, sight of blood or medical procedures, heavy exercise, and rising or changing positions abruptly [6]. From episode to episode the symptoms will vary and can be estimated by a simple risk score [7]. The hypotension is thought to be caused by a reduction in peripheral sympathetic neural outflow, which leads to venous pooling and vasodepression. Yet, the exact central neurophysiology is not known [8]. The majority of cases of syncope in young people are not typically associated with risk of mortality [9], but physical and psychosocial function can be seriously impaired in patients suffering from recurrent syncope [10].

A number of treatments have been proposed for NCS [e.g., 1,2], but only one focused review of therapies with structured recommendations was found. Most patients can be managed conservatively and the recommended treatments included liberalized fluid and salt intake, patients being taught counter-pressure maneuvers and $\mathrm{Mi}$ dodrine to manage hypotension. Beta blockers, specific serotonin reuptake inhibitors (SSRI), fludrocortisones, and pacemakers have had mixed results, and were therefore discouraged for use [8].

Specifically for SSRI treatment, there were only two 
randomized double-bind placebo-controlled study found using paroxetine in 68 patients (mean age 44.7, SD = 16.5) with recurrent syncope and positive head-up tilt test [11] and 41 young patients (aged less than 30 years old) with recurrent syncope [12]. The conclusion in both studies was that paroxetine significantly improved symptoms of patients with vasovagal syncope not responding well with more traditional medications and treatments. Of note, although paroxetine treatment was significantly superior to placebo, more than one-third of patients remained symptomatic. Another randomized, placebo-controlled study using fluoxetine, propranolol, and placebo showed no differences between treatment groups [13], therefore, it is possible that not all SSRI are effective in treating recurrent vasovagal syncope. Additionally others have argued for the importance of addressing the psychological distress in these patients [14, 15]. The purpose of this paper is to present a case of a young woman who was treated with a combination of paroxetine and cognitive/behavioral strategies to address her recurrent NCS symptoms.

\section{METHODS}

\section{Case Report}

While the patient was in high school at the age of 17 she developed feelings of malaise, what she thought was fever and chills, but was not febrile. She had periods of feelings of weakness and fatigue, experienced diaphoresis, diminished appetite, oversleeping, and hypotension. During high school, she had one period of syncope for approximately one minute that occurred after a band practice. Since that time she had not experienced true syncope, but rather ataxia, nausea, extreme fatigue, and vertigo where she would need to lie on the ground to stop the vertigo. She also was placed on Ethinyl Estradiol and Norgestimate (Ortho Tri-Cyclen) for dysmenorrhea.

The syncope symptoms continued and she was seen by an internist. At one point her symptoms were suggestive of malaria since she had been in Honduras and upon returning had developed uticaria, which later was determined to be eczema. Blood results ruled out malaria, mononucleosis, West Nile virus, and Lyme disease. She then was tested for demyelinating disease by an MRI brain scan, which provided evidence of a normal brain MRI, without and with contrast. She further was tested with a two-dimensional transthoracic echocardiogram (ECG) with M-mode and color flow Doppler. The results were deemed normal. The diagnosis of NCS was suggested when a tilt test was performed where her resting heart rate and blood pressure were normal. Her health record indicated, "She was maintained in an 80-degree upright tilt position and within 15 minutes, she devel- oped sinus tachycardia with a sudden loss of palpable blood pressure with near syncope." She was then returned to a supine position where her abnormal vital signs resolved.

The patient was treated conservatively and was encouraged to increase her salt intake, use counter-pressure measures, and rest when she experienced fatigue and dizziness. According to her health record, she was started on metoprolol $25 \mathrm{mg}$. a beta-blocker with mixed results in several studies (8). However, her response was blood pressure of 98/50 and pulse 64, so the treating physician was reluctant to increase the dosage due to the borderline blood pressure and heart rate. The beta-blocker was discontinued since there was not a significant decrease of her syncope symptoms. Because of her history of depressive symptoms she was started on $14.5 \mathrm{mg}$. of sertraline to increase to $25 \mathrm{mg}$., however immediately after taking one dosage of sertraline she had a serious episode with extreme fatigue, near syncope and disturbing visual images so the treating physician was reluctant to have her continue with the medication. Because of her history of depression and treatment strategies had not decreased the number of episodes; the patient, at age 20, was referred to the Depression Center at University of Michigan by her sister, a nurse practitioner.

In her evaluation, she reported that she first experienced depressive symptoms at the age of 14, but while in her junior year of high school (age of 17) she had depressive symptoms including depressed mood, feelings of fatigue, trouble concentrating, insomnia, feelings of worthlessness, overeating, but not gaining weight, and thoughts of suicide. She had approximately a week of feeling suicidal and had developed a plan for suicide by causing a car accident. She had made a specific plan, but a friend called her and asked her how she was doing. At that moment she realized she did not want to die. The symptoms diminished and she did not seek treatment nor diagnosed with depression at the time. At evaluation, she described her symptoms of depression as mild depressed mood, anhedonia, loss of appetite, trouble concentrating, feeling restless, moderate insomnia, and lack of energy. She denied suicidal ideations. She scored 10 on the Patient Health Questionnaire (PHQ-9) indicating moderate depression, and 19 on the clinician administered Hamilton Rating Scale for Depression (HAM-D). She met criteria for a diagnosis of Major Depressive Disorder (MDD). Moreover, she scored 11 on the Sleep Assessment Questionnaire (SAQ), indicating a potential sleep problem. Her score on the Penn State Worry Questionnaire (PSWQ) was 33, indicating likely sub-clinical anxiety.

Most concerning was her description of vivid visual images where her heart would pound, feelings of panic, and where she described the images as "hallucinations." 
She became tearful as she described that these visual images could occur 3 or 4 times a week and could last up to 20 minutes but often shorter, where she would visualize "dead babies" and see "killing" herself. She noted that after these episodes it would often take her a week or more to recover since she experienced a depressed mood, anhedonia, and extreme fatigue. Since her depression in high school she also described experiences where she "wanted to crawl in small spaces." As examples, she sat in a closet or under a desk. This was especially noteworthy in her junior year at high school where she was entering the closet or under the desk about 2 or 3 times a week. During college she would get the feeling, but acted on it only a few times.

\section{RESULTS}

Because of the possibility that the previous SSRI might have triggered the disturbing visual images, the patient was provided scientific evidence $[11,12]$ that paroxetine was promising as an effective treatment for depression in NCS. The theoretical mechanism of how paroxetine would work in her brain was explained with a drawing and the typical side effects of the medication also were presented. Since the patient appeared to be sensitive to medications, she was started at only $5 \mathrm{mg}$. of paroxetine each day in the morning. Also, she was recommended to start $2000 \mathrm{mg}$. of omega-3 fatty acid based on the evidence it can also help to boost mood and vagal tone [16]. She was recommended to purchase omega-3 that contained $1000 \mathrm{mg}$. of eicosapentaenoic acid (EPA) and docosahexaenoic acid (DHA), where EPA is considerd to be the ingredient with the most therapeutic effect.

She was educated about the physiology of NCS in lay terms. After several days after starting treatment, she was called at home to check if she had any side effects or any disturbing images. She only experienced a slight headache and nausea for a day from the paroxetine. After one week, her PHQ-9 score decreased to 8 indicating mild depressive symptoms, which was lower than her initial evaluation. Attributable to a slight decrease in depressive symptoms was the several cognitive/behavioral strategies she was given. She noted that when she would experience fatigue she had a tendency to get increasingly concerned that another episode would occur. She was taught thought-stopping and thought-substitution. We identified an experience that gave her "nothing but warm feelings" and she identified the experience as the moment when her fiancé asked her to marry him. Therefore, during the first week of treatment, if she experienced fatigue or weakness, she used this imagery strategy reporting that it was helpful.

Since the patient had minimal side-effects from paroxetine her dosage was increased to $10 \mathrm{mg}$. In the session she identified a pattern that she noticed when she drove from her college to see her fiancé (approximately a 3-hour drive). She would tense grip the steering wheel and would experience fatigue and weakness after the drive. She was taught deep-breathing by taking a deep breath and letting it out over a 10 -second period and progressive relaxation by tensing a muscle group and relax it over a 10 -second period. Since she had to hold the steering wheel, she was instructed to relax one arm and hand at a time and shake out the tension about every 15 minutes while driving. She also was encouraged to use progressive relaxation when she occasionally would experience early insomnia. At the next session a week later, she reported that these strategies were helpful and her PHQ-9 score was reduced to 4 indicating minimal depressive symptoms. She also noted that the treatment had helped with her dysmenorrhea symptoms.

After six-months from initial treatment the patient had no syncope and depressive symptoms. Most indicative of her progress was that several weeks before her appointment she was the Maiden-of-Honor for her sister's wedding. Her family was concerned that she would not be able to stand through the entire ceremony. She reported that she had no periods of weakness or vertigo and danced late into the evening. Her fiancé also reported that she was able to play a full set of tennis where before she would need to take breaks to play.

\section{DISCUSSION}

There is limited evidence as to the most effective treatments for NCS. Some conservative strategies have been suggested $[1,2,5,8]$, but only one research team provided randomized controlled studies [11,12] suggesting paroxetine as an effective treatment for at least some patients with NCS. Yet, another randomized control study using fluoxetine did not demonstrate effectiveness [13]. This conflicting evidence led some researchers [8] to conclude that SSRIs are not effective in treating NCS. Also, one study found that paroxetine did not prevent the presyncope associated with lower body negative pressure in healthy volunteers [17]. These mixed results may suggest that SSRIs are not effective in individuals with NCS, yet the specific use of paroxetine with patients having NCS and depression may be more effective than treating patients with other SSRIs.

There is evidence that co-morbid mood disorders are present in a significant proportion of patients with syncope [18]. Studies have shown that a combination of antidepressants and psychotherapy are more effective at achieving remission in chronic depression that either treatment alone [e.g., 19]. In this case report a multifaceted approach was used to address the NCS and depressive symptoms. Using paroxetine in conjunction with omega-3 fatty acids, counter-pressure techniques, and cognitive/behavioral strategies to address the distre- 
ssing events $[14,15]$ was ultimately successful. The use of progressive relaxation, imagery, sleep strategies, and exercise helped with the life altering experience. Prior to treatment, the patient often experienced fatigue, weakness, vertigo, disturbing images, and depressed mood. Now, the patient is able to take part in normal and productive activities and not have concern about NCS and the concomitant symptoms. This report suggests that a combination treatment may be most effective to achieve remission in patients suffering from NCS, particularly in the context of depressive symptoms, and that these strategies should be evaluated in future randomized clinical trials.

\section{ACKNOWLEDGEMENTS}

This article was made available as Open Access with the support of the University of Michigan COPE Fund, http://lib.umich.edu/cope.

\section{REFERENCES}

[1] Grubb, B.P. and McMann, M.C. (2001) The fainting phenomenon: Understanding why people faint and what can be done about it. Futura, New York.

[2] Neurocardiogenic syncope (2011). http://www.dinet.org/NCS/ncs.htm

[3] Savage, D.D., Corwin, L., McGee, D.L., Kannel, W.B. and Wolf, P.A. (1985) Epidemiologic features of isolated syncope: The Framingham Study. Stroke, 16, 626-629. doi:10.1161/01.STR.16.4.626

[4] Chen, L., Chen, M.H., Larson, M.G., Evans, J., Benjamin, E.J. and Levy, D. (2000) Risk factors for syncope in a community based sample (the framingham heart study). American Journal of Cardiology, 85, 1189-1193. doi:10.1016/S0002-9149(00)00726-8

[5] Deering, A (2007) Cheltenham syncope clinic. http://www.sycope.co.uk/

[6] Alboni, P., Brignole, M., Menozzi, C., Raviele, A., DelRosso, A., Dinelli, M., Soluno, A. and Bottoni, N. (2001) Diagnostic value of history in patients with syncope with or without heart disease. Journal of American College of Cardiology, 37, 1921-1928. doi:10.1016/S0735-1097(01)01241-4

[7] Aydin, M.A., Maas, R., Mortensen, K., Steinig, T., Kleem, H., Risius, T., Meinertz, T., Willems, S., Morillo, C.A. and Ventura, R. (2008) Predicting recurrence of vasovagal syncope: A simple risk score for the clinical routine. Journal of Cardiovascular Electrophysiology, 20, 416-421. doi:10.1111/j.1540-8167.2008.01352.x

[8] Kuriachan, V., Sheldon, R.S. and Platonov, M. (2008) Evidence-based treatment for vasovagal syncope. Heart Rhythm, 5, 1609-1614. doi:10.1016/j.hrthm.2008.08.023

[9] Bolles, J.A., Gentlesk, P.J., Lewis, P.C., Ross, R.D. and Eckart, R.E. (2010) Resource utilization in the manage- ment of young patients with syncope in a combat theater of operations. Military Medicine, 175, 324-327.

[10] Linzer, M., Pontinon, M. and Gold, D.T. (1991) Impairment of physical and psychosocial health in recurrent syncope. Journal of Clinical Epidemiology, 44, 10371043. doi:10.1016/0895-4356(91)90005-T

[11] Di Girolamo, E., Di Iorio, C., Sabatini, P., Leonzio, L., Barbone, C. and Barsotti, A. (1999) Effects of paroxetine hydrochloride, a selective serotonin reuptake inhibitor, on refractory vasovagal syncope: A randomized, double-bind, placebo-controlled study. Journal of the American College of Cardiology, 33, 1227-1230. doi:10.1016/S0735-1097(98)00694-9

[12] Di Girolamo, E., Di Iorio, C., Leonzio, L., Sabatini, P., Ranalli, G. and Barsotti, A. (1999) Effects of paroxetine in the treatment of refractory vasovagal syncope in young patients. Giornale Italiano Di Cardiologia, 29, 1472-1477.

[13] Foglia-Manzillo, G., Giada, Fr., Gaggioli, G., et al. (2004) Effacacy of tilt training in the treatment of neutrallymediated syncope. A randomized study. Europace, 6, 199204. doi:10.1016/j.eupc.2004.01.002

[14] D’Antono, B.D., Dupuis, G., St-Jean, K., Levesque, K., Nadeau, R., Guerra, P., Thibault, B. and Kus, T. (2009) Prospective evaluation of psychological distress and psychiatric morbidity in recurrent vasovagal and unexplained syncope. Journal of Psychosomatic Research, 67, 213-222. doi:10.1016/j.jpsychores.2009.03.012

[15] Gracie, J., Newton, J. L., Norton, M., Baker, C. and Reeston, M. (2006) The role of psychological factors in response to treatment in neurocardiogenic (vasovagal) syncope. Journal of the Working Groups on Cardiac Pacing, Arrhythmias, and Cardiace Cellular Electrophysiology of the European Society of Cardiology, 8, 636-643. doi:10.1093/europace/eul073

[16] Kiecolt-Glaser, J.K. (2010) Stress, food, and inflamemation: Psychoneuroimmunology and nutrition at the cutting edge. Psychosomatic Medicine, 72, 365-369. doi:10.1097/PSY.0b013e3181dbf489

[17] Takata, T.S., Wasmund, S.L., Smith, M.L., Li, J.M., Joglar, J.A., Banks, K., Kowal, R.C., Page, R.L. and Hamdan, M.H. (2002) Serotonin reuptake inhibitor (Paxil) does not prevent the vasovagal reaction associated with carotid sinus massage and/or lower body negative pressure in healthy volunteers. Circulation, 106, 1500-1504. doi:10.1161/01.CIR.0000029748.94931.96

[18] Linzer, M., Varia, I., Pontinen, M., et al. (1992) Medically unexplained syncope: Relationship to psychiatry illness. American Journal of Medicine, 92, S18-S25. doi:10.1016/0002-9343(92)90132-U

[19] Keller, M.B., McCullough, J.P., Klein, D.N., et al. (2002). A comparison of nefrzodone, the cognitive behavioral analysis system of psychotherapy, and their combination for the treatment of chronic depression. New England Journal of Medicine, 342, 1462-1470. doi:10.1056/NEJM200005183422001 